\title{
The Extended Marine Underwater Environment Database and Baseline Evaluations
}

\author{
Muwei Jian ${ }^{1,3 *}$, Qiang $Q i^{2}$, Hui Y ${ }^{3}$, Junyu Dong ${ }^{4}$, Chaoran Cui ${ }^{1}$, Xiushan Nie ${ }^{1}$, Huaxiang Zhang ${ }^{5}$, \\ Yilong $\mathrm{Yin}^{6}$, Kin-Man Lam ${ }^{7 *}$ \\ ${ }^{1}$ School of Computer Science and Technology, Shandong University of Finance and Economics, Jinan, China. \\ ${ }^{2}$ Department of Information Centre, Zibo Municipal Party School, Communist Party of China, Zibo, China. \\ ${ }^{3}$ School of Creative Technologies, University of Portsmouth, Portsmouth, UK. \\ ${ }^{4}$ Department of Computer Science and Technology, Ocean University of China, Qingdao, China. \\ ${ }^{5}$ School of Information Science and Engineering, Shandong Normal University, Jinan, China. \\ ${ }^{6}$ School of Software Engineering, Shandong University, Jinan, China. \\ ${ }^{7}$ Centre for Signal Processing, Department of Electronic and Information Engineering, \\ The Hong Kong Polytechnic University, Hung Hom, Kowloon, Hong Kong \\ *(Corresponding author) E-mail: jianmuweihk@163.com
}

\begin{abstract}
Images captured in underwater environments usually exhibit complex illuminations, severe turbidity of water, and often display objects with large varieties in pose and spatial location, etc., which cause challenges to underwater vision research. In this paper, an extended underwater image database for salient-object detection or saliency detection is introduced. This database is called the Marine Underwater Environment Database (MUED), which contains 8600 underwater images of 430 individual groups of conspicuous objects with complex backgrounds, multiple salient objects, and complicated variations in pose, spatial location, illumination, turbidity of water, etc. The publicly available MUED provides researchers in relevant industrial and academic fields with underwater images under different types of variations. Manually labeled ground-truth information is also included in the database, so as to facilitate the research on more applicable and robust methods for both underwater image processing and underwater computer vision. The scale, accuracy, diversity, and background structure of MUED can not only be widely used to assess and evaluate the performance of the state-of-the-art salient-object detection and saliency-detection algorithms for general images, but also particularly benefit the development of underwater vision technology and offer unparalleled opportunities to researchers in the underwater vision community and beyond.
\end{abstract}

Keywords: benchmark; underwater vision; underwater image database; saliency detection. 


\section{Introduction}

Recently, autonomous underwater navigation has become a hot area of research with broad commercial and military applications. Hence, underwater image/video processing has drawn wide attention. On the one hand, projects like North-East Pacific Undersea Networked Experiments (NEPTUNE) sub-sea observatories suffer from an every-increasing difficulty and the time requirement for the extraction of useful information as their underwater video/image databases grow massively. On the other hand, the exploration and development of marine resources takes place all over the world. Thus, the precise detection of abnormal and salient objects in underwater environments is one of the primary and essential issues in underwater navigation research nowadays. Underwater saliency detection, serving as a pre-processing step like conventional visual saliency detection, aims to identify the most informative location of objects in underwater images and greatly benefits many practical computervision applications in similarity search/matching [1, 2, 4], object retargeting [3], automatic image cropping [5], scene classification [6], moving object detection [43], and motion detection [44, 45]. This detection can not only efficiently focus on the interesting underwater image regions or objects, but also particularly benefits the development of underwater vision technology in the future.

Although significant progress has been made in last two decades, visual saliency detection remains a challenging issue $[9,11,15,19,24,34,37,40]$ due to the interrelated and mutually influenced interactions in the underwater scenarios, such as complicated illumination conditions, low-contrast objects under complex background, light absorbing and scattering, severe turbidity of water, etc. Therefore, modeling the saliency-detection mechanism in underwater scenes is a tough and challenging task. In particular, although there exists a data set of turbid underwater images for evaluating imagerestoration methods [42], publicly available underwater databases can seldom be used for underwater saliency detection and only limited research has been carried out to investigate how to tackle underwater salient-object detection [28, 29].

Good benchmark datasets will accelerate and promote the development of related research fields [54]. In this paper, to extend saliency detection into the underwater vision domain, an extended underwater image database based on the OUC-VISION database [33], called the Marine Underwater Environment Database (MUED), is constructed and presented. This database contains underwater images with pose variations (with frontal, opposite, left, and right views of each individual underwater object), changes of different spatial location (underwater object located at the top-left corner, the top-right corner, the center, the bottom-left corner, and the bottom-right corner), complicated illumination conditions, varying 
degrees of turbidity, complex background and multiple-salient objects, in a single uniform database. The currently released underwater image data set contains 8600 images, 430 groups of underwater subjects under different conditions. Furthermore, we will also describe the design, collection, labeling, and categorization of the samples for the database in detail. The advantages of the designed underwater image database are that there are a large number of images for each distinct subject, and the images are categorized according to the controlled variations of the different situations. In our experiments, we have also compared and evaluated the performance of several state-of-the-art saliency-detection methods on this publicly available underwater database.

The main contributions of this paper are summarized as follows:

$>$ To the best of our knowledge, no underwater database for saliency detection and assessment is publicly available. To extend saliency detection to the underwater vision domain, a diverse underwater image database is constructed and presented.

$>$ This released underwater image database can be used to identify the strengths and weaknesses of the existing state-of-the-art saliency-detection algorithms for underwater images. The experiments with algorithms on the underwater image database provide reference-evaluation results for researchers working on underwater computer vision.

$>$ This database can not only offer unparalleled opportunities to researchers in the underwater vision community and beyond, but also particularly benefit the development of underwater vision technology in the future.

A preliminary conference version of the released database was published in [33]. In contrast to the previous work in [33], which contained 4400 underwater images of 220 individual objects, the new MUED includes 4200 more underwater images of 210 conspicuous underwater objects. Furthermore, this database also considers complex underwater background variations, underwater floating debris, and multiple salient objects, so as to construct a larger and more comprehensive data set. We also provide baseline evaluation results to researchers in the underwater vision community, to facilitate the research on more applicable and robust methods for both underwater image processing and underwater computer vision.

The remainder of this paper is organized as follows. In Section 2, we will introduce the related work on saliency detection and underwater salient-object detection. In Section 3, we introduce the imaging set-up and the lighting system for capturing underwater images. Then, the design and the contents of the MUED are presented in Section 4. In Section 5, the designed database is described in detail. The 
performance of several state-of-the-art saliency-detection methods is evaluated on the MUED in Section 6. Section 7 describes how to obtain the MUED. Finally, the paper closes with a conclusion and discussion in Section 8.

\section{Related Work}

Visual saliency detection, aiming at locating salient regions or conspicuous objects in an image, has recently drawn much attention. It has also been extensively studied in both the fields of image processing and computer vision [7]. Practical applications of saliency detection include image segmentation, automatic image retargeting, visual tracking, object recognition, etc. Generally, saliencydetection computational models fit into two categories: those based on the bottom-up model and those based on the top-down model. The bottom-up approaches [1, 12-29] consider low-level stimuli, such as color, contrast, location and texture, and are usually data-driven. The top-down approaches [30, 31], which use supervised methods with high-level cues, are often task-dependent and goal-driven.

Over the past decades, most of the bottom-up computational schemes simulating primate perceptual abilities uses low-level visual features to estimate image saliency. The pioneering model, which simulates the Human Visual System (HVS) for visual saliency detection, utilized a center-surround operator to compute saliency across three low-level cues [7]. Later, lots of bottom-up salient-object detection methods were developed, which are based on low-level handcrafted features, such as image contrast [8], pattern distinctness [9], texture [10], etc. In [11], a novel graph-based method was proposed to estimate the saliency map in order to make salient-object detection more precise. However, the graphbased method usually generates low-resolution saliency maps. Thus, a spectral residual-based saliencydetection method was proposed by Hou and Zhang in [12]. These early methods found it hard to handle cases with complex image scenes or backgrounds. To soften this problem and acquire accurate salient regions, Ma et al. [13] presented a local contrast-based method for saliency detection, which increases the accuracy of saliency detection for the images with complicated background. In [14], a special colorappearance method, based on a non-parametric low-level vision model, was proposed for saliency detection. In [15], a visual-attention-aware model was proposed to mimic the HVS for salient-object detection. Song et al. [16] proposed a RGBD co-saliency method, which uses bagging-based clustering to detect salient objects. In $[17,18]$, a saliency-detection method was devised, which utilizes both visual-directional stimuli and two low-level object cues. In order to take object boundary information into account, a novel hierarchical model incorporating probabilistic object boundaries was presented for 
salient-object detection in [19]. Recently, deep learning has delivered superior performance in the saliency-detection task. Wang et al. [24] proposed a deep neural network, which integrates local estimation and global search, for saliency detection. In [25], an end-to-end deep contrast network was proposed to predict the saliency degree of each superpixel. However, these deep-learning-based saliency-detection models contain fully connected layers, which are computationally expensive. In addition, the fully connected layers drop the spatial information of input images. To alleviate these issues, Li et al. [26] proposed a multi-task deep model based on a fully convolutional neural network with global input and global output for saliency detection. Later, in order to improve the accuracy of the saliency results and address the problem that salient objects may appear in a low-contrast background, Hou et al. [27] proposed a novel saliency-detection approach by integrating short connections and the skip-layer structures within the Holistically-Nested Edge Detector (HED) architecture. Zhang et al. [28] designed a novel model based on convolutional network with fully connected output layer, which learns deep uncertain convolutional features (UCF), for accurate salient-object detection. Pang et al. [45] presented a $\mathrm{CiC}$ (convolution in convolution) method, which has a stronger capability for feature representation and hence can be used to extract the image's saliency.

Compared to the bottom-up saliency-detection models, which utilize low-level visual features in images, another saliency-detection mechanism is the top-down model. It is a learning-based framework, and relies on high-level cues and prior knowledge. In [20], an efficient top-down saliency-detection model based on a Bayesian framework, which incorporates the object-appearance cues and scene context, was proposed to estimate saliency maps of input images. Later, a top-down visual saliency model, which combines dictionary learning and the Conditional Random Field (CRF), was proposed in [21]. Murabito et al. [22] presented an approach for top-down saliency detection guided by visual classification. In [23], a novel locate-by exemplar top-down framework, which incorporates deep association and background discrimination, was proposed to detect the saliency of images.

Although many saliency-detection models have been proposed, their performance is still unsatisfactory when the images contain complex backgrounds or low-contrast objects, as is the case for images captured underwater. In particular, successfully applying a saliency-detection mechanism to underwater scenes is a difficult and challenging task, owing mainly to the following reasons. Images captured with underwater objects are usually affected by variations of complicated illumination conditions, low-contrast object appearances, turbidity of water, light absorbing and scattering, lack of color information, etc., which are all challenges to the state-of-the-art saliency-detection models. 
Currently, only limited research has been carried out to investigate how to tackle underwater salientobject detection. In [29], a vision-based system for underwater object detection was presented to detect objects on the sea bed. Zhang et al. [30] proposed a method for underwater image detection based on the discrete fractional Brownian random field. In [31], an underwater target detection method was proposed, which combines active polarization imaging and optical correlation-based approaches. Wang et al. [32] presented a saliency-based adaptive object-extraction algorithm for underwater images. Recently, a framework [18] based on the Quaternionic Distance Weber Local Descriptor (QDWD) and low-level priors of object cues was proposed for natural image saliency detection. Firstly, QDWD is used to represent the directional cues in an image. Then, two low-level priors, namely the Convex-Hull-Based center and color contrast cues of the natural image, are utilized and fused as an object-level cue. Finally, by combining QDWD with object cues, a reliable saliency map of the image can be computed.

The complex underwater environment usually induces a lack of color information of the objects as well as variations of complicated lighting conditions in the captures images, we designed a new method in [41] by integrating the Quaternionic Distance Based Weber Descriptor and pattern distinctness with local contrast to estimate the underwater image saliency. In contrast to the former work in [18], our algorithm proposed in [41] incorporates the quaternion number system and principal components analysis (PCA) simultaneously, so as to achieve superior performance for underwater image saliency detection. In addition, we also adopt pattern distinctness and local contrast in [41] to respectively reduce the influence of complicated lighting conditions and dispose of the low-contrast underwater objects by analyzing the intrinsic properties of underwater images, which can detect almost the entire underwater salient objects with obvious boundaries and less background pixels.

However, to the best of our knowledge, no public underwater database for underwater saliency detection is available, which is perhaps the primary reason for attaining little improvement in the area of underwater saliency detection. To address this problem, in this paper, we introduce a diverse underwater image database, which can not only be widely used to assess and evaluate the performance of the stateof-the-art saliency-detection algorithms for general images, but also particularly benefits the development of underwater image processing [46-48], underwater image enhancement/restoration algorithms [49-52], and underwater vision technology in the future.

\section{Underwater Imaging Set-Up}


In order to mimic the environment of the ocean floor and capture underwater images with different variations in pose, spatial location, illumination, turbidity, background and the number of salient objects, we set up a special cube pool with the dimensions $1.5 \mathrm{~m} \times 1.5 \mathrm{~m} \times 1.5 \mathrm{~m}$. The cube pool is full of water, with sand and weed at its bottom, so as to simulate the ocean floor. The necessary facilities are installed in the underwater photographic room in the OUC-VISION laboratory, including a camera system and a lighting system. Fig. 1 illustrates the imaging set-up of the camera and the lighting system for capturing underwater images. The details are described in the following sections.

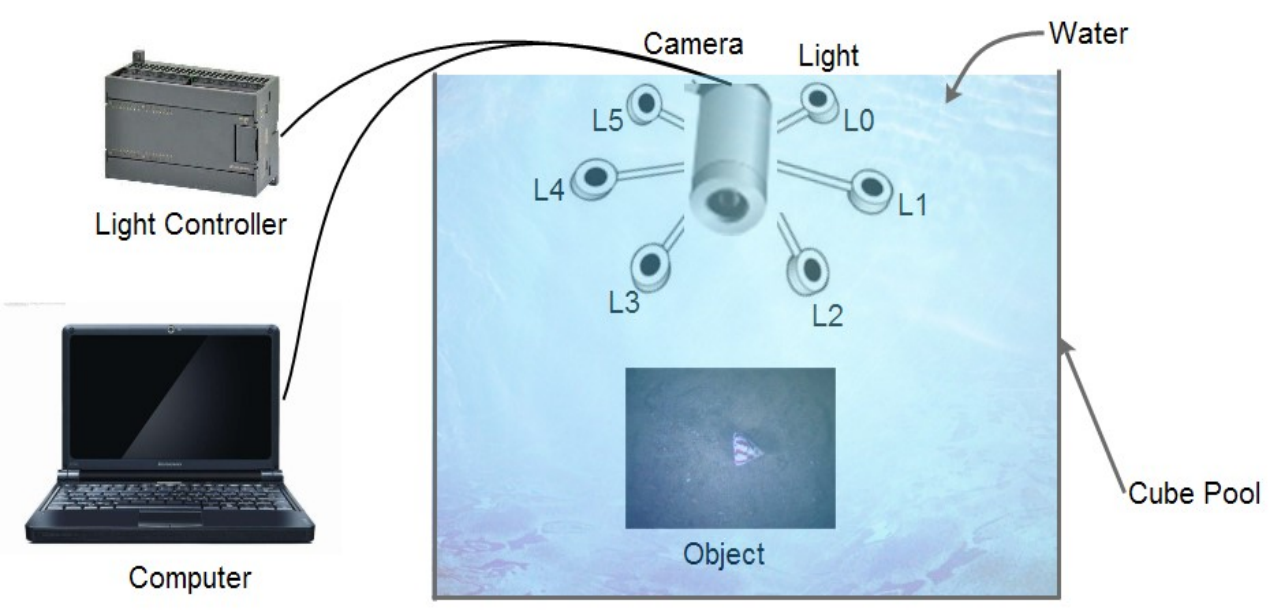

Fig. 1. The sketch map of the imaging set-up for the camera and lighting system.

\subsection{Camera system}

In our underwater photographic room, a camera system is made up of an MV-GE500C camera and a computer. The underwater camera MV-GE500C has a resolution of $2592 \times 1944$ pixels. The camera is connected to the computer through a USB interface. We developed a special software system to control the camera and capture the images in a shot. Once the capture button is clicked, the software will direct the camera to collect the underwater images of an object and store the images in its memory device.

To capture the images of a whole object, the seabed surface and camera are fixed, and the object is placed completely within the field of view of the camera. Furthermore, the object is moved to different positions, i.e. the center and the four corners of the images, when images are being captured.

\subsection{Lighting System}


A special lighting system, with six high-power Light Emitting Diodes (LEDs) lights, is used to simulate the underwater ambient illumination. The six LEDs lights are mounted on a hexagon with the same distance apart, as shown in Fig. 1. They are labeled as L0-L5, in their clockwise direction, with $60^{\circ}$ separation between adjacent lights. Each light has its own switch, so we can control them separately. Fig. 1 shows a sketch map of the lighting set-up.

In our photographic room, we used the symmetrical lights of L0, L2, L4 to capture underwater images to mimic different illuminations. By combining the three LED lights, different lighting conditions can be simulated. In our implementation, the illumination variations are not strictly controlled, but these lighting variations can be easily distinguished.

\section{Design of the Marine Underwater Environment Database}

Six variations, namely pose, spatial location, illumination, turbidity, background, and the number of salient objects, are considered simultaneously in the construction of the MUED. The salient objects in the images were also labelled by different people, so as to provide ground truths for evaluation and assessment purposes. The following sections will describe the six variations and the labeling process in detail.

\subsection{Pose Variations}

To capture underwater images with various poses of an object, we consider four sides (the front, the opposite, the left, and the right) of each underwater object. In each side, an underwater image is collected from the camera in one shot. Thus, a total of four underwater images for each subject are captured. Fig. 2 shows an example of the four sides of an underwater object.
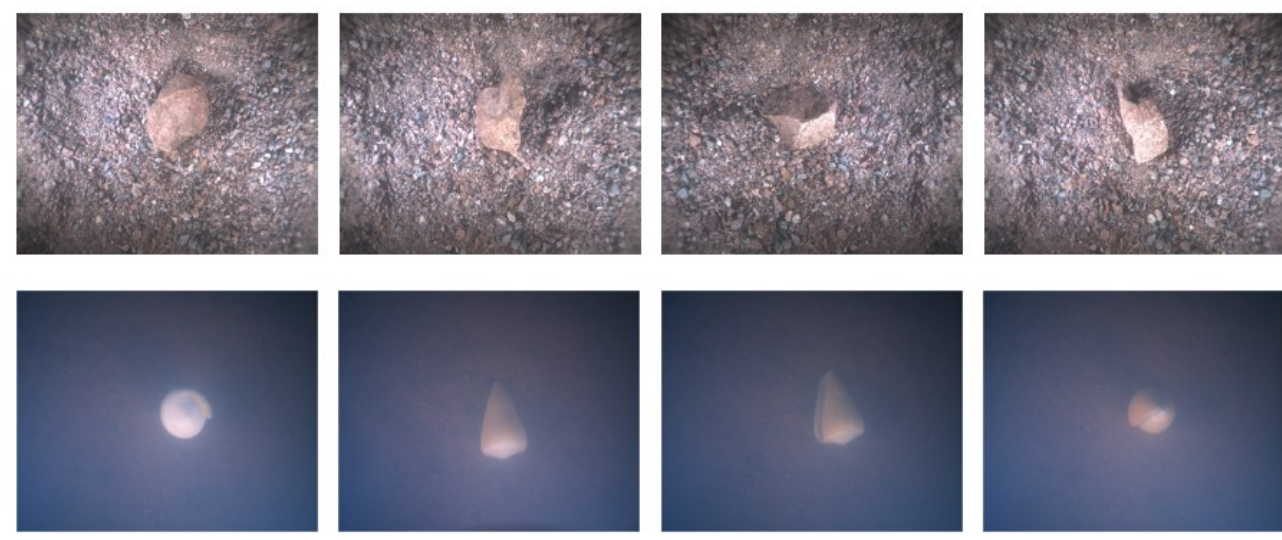

(a)

(b)

(c)

(d)

Fig. 2. Two typical underwater objects at different poses: (a) front-, (b) opposite-, (c) left-, and (d) rightside, of an individual underwater object. 


\subsection{Spatial Location Variations}

In order to reflect the variations in terms of spatial locations in our presented underwater database, each underwater object is placed at five different locations, including the top-left corner, the top-right corner, the center, the bottom-left corner, and the bottom-right corner of the image. Therefore, for each of the poses, five underwater images, with the object at different spatial locations, are captured. Fig. 3 shows the five images of an underwater object with the same pose.
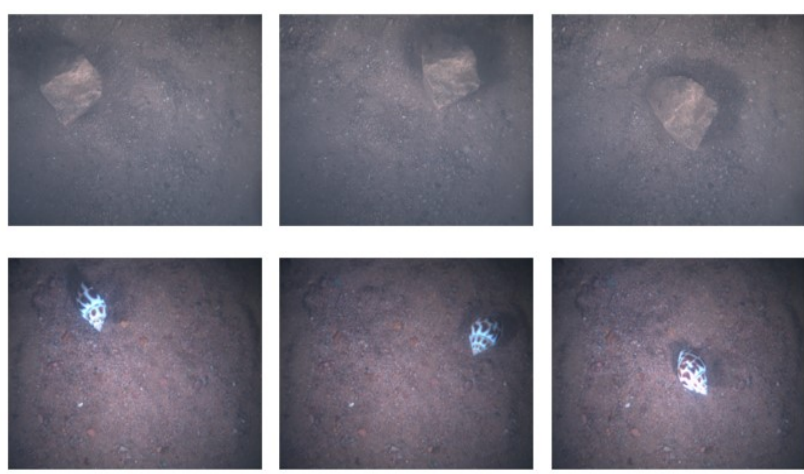

(a)

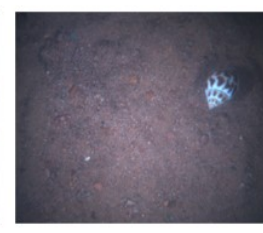

(b)

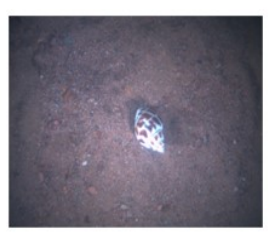

(c)
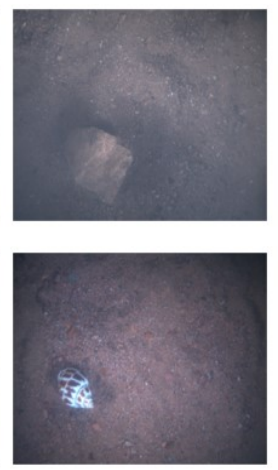

(d)
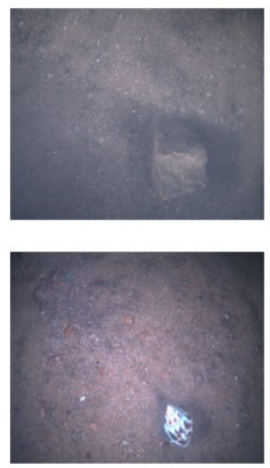

(e)

Fig. 3. The spatial-location variations of two typical underwater objects. The object is placed at (a) the top-left corner, (b) the top-right corner, (c) the center, (d) the bottom-left corner, and (e) the bottom-right corner, of the respective images.

Each object is captured with four different poses (the front, the opposite, the left, and the right views of each underwater object) and at five different locations. Therefore, each underwater object is captured with twenty images when both the pose and spatial-location variations are considered. Twenty example images of an underwater object, with four poses and five spatial locations, are illustrated in Fig. 4. 

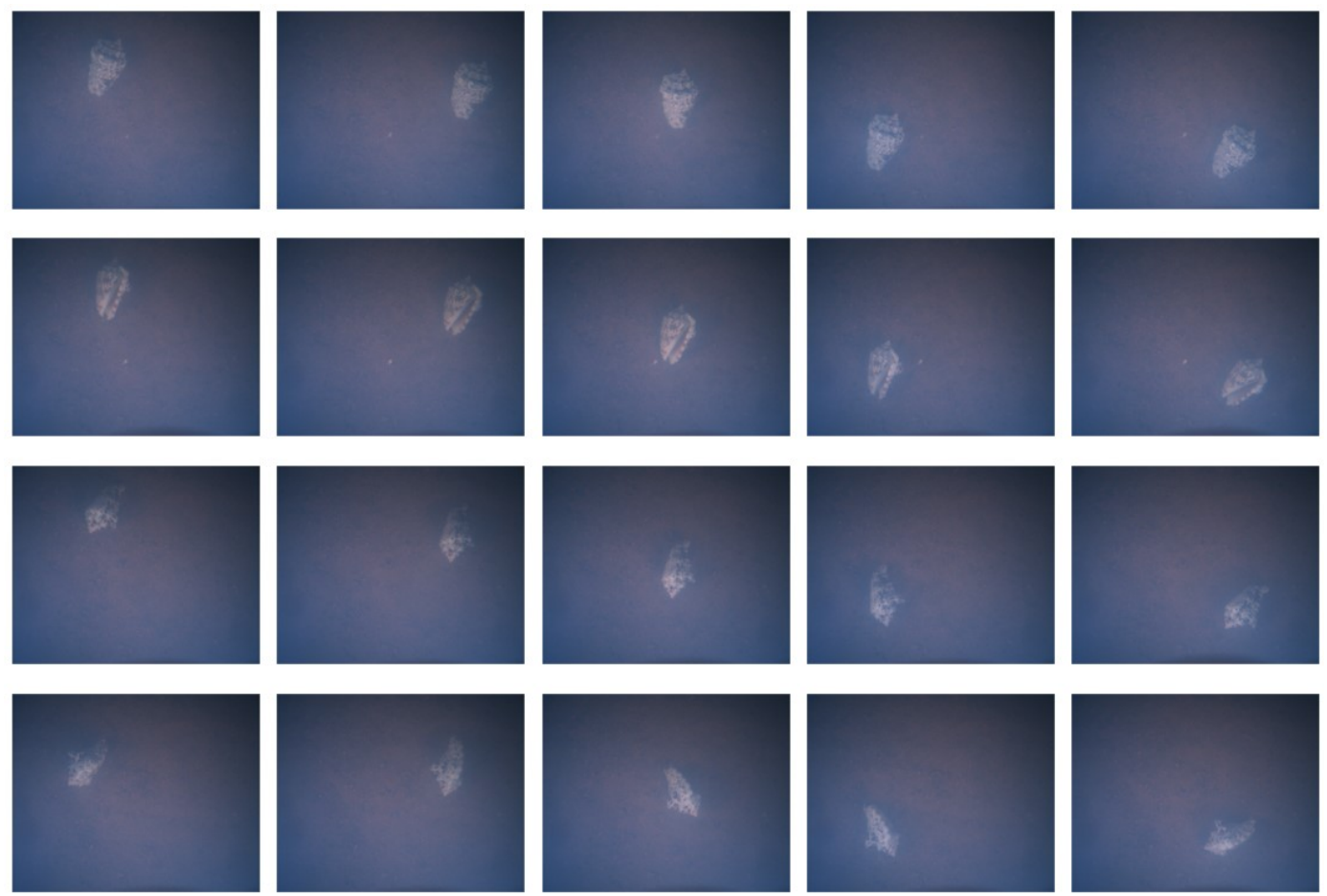

Fig. 4. An underwater object in four poses and five spatial locations.

\subsection{Lighting Variations}

As described in Section 3.2, the L0, L2 and L4 LED lights in the lighting system are controlled to simulate different underwater lighting conditions. During the capture of an underwater image, four different illumination conditions are generated, by turning on all the three LED lights, two of the LED lights, one LED light, and turning off all the three LED lights, respectively. Fig. 5 shows some sample underwater images under the four different lighting conditions.
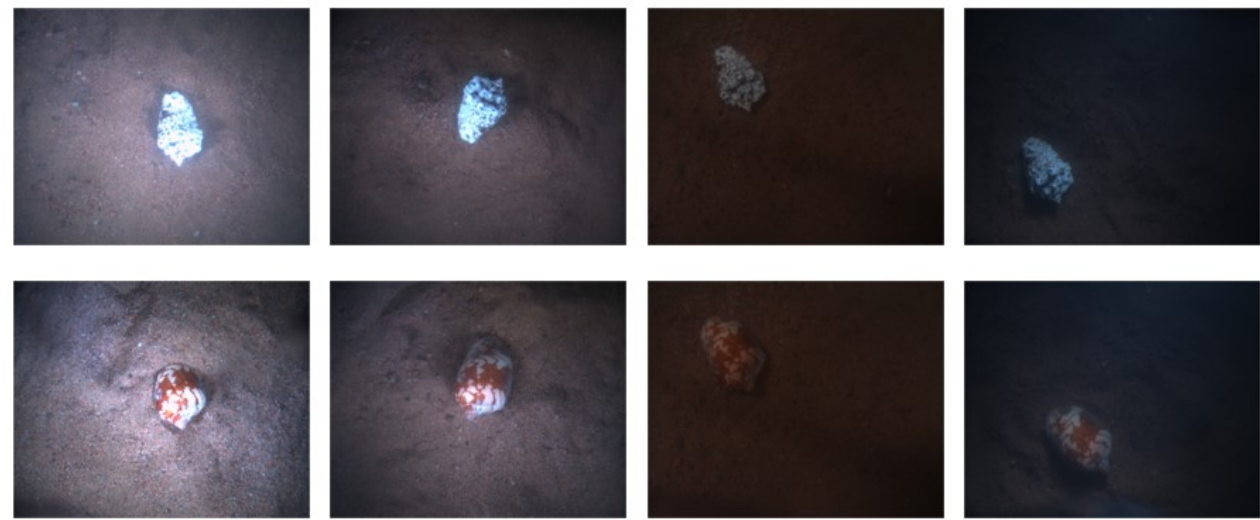

Fig. 5. The underwater images captured with four different lighting variations. 


\subsection{Turbidity Variations}

The variations in water turbidity are an important factor influencing saliency detection of underwater images. The turbidity is subject to change in the ocean in real applications. In order to evaluate its effect, three degrees of variations in water-turbidity, i.e. limpidity, medium, and high, are considered, as illustrated in Fig. 6. We change the water turbidity by adding soil and milk to the cube pool. From Fig. 6, it can be seen that the appearances of the underwater images are highly dependent on the turbidity of the water. When the turbidity of the water is stronger, the images become more blurred and unclear in the complex underwater environment.
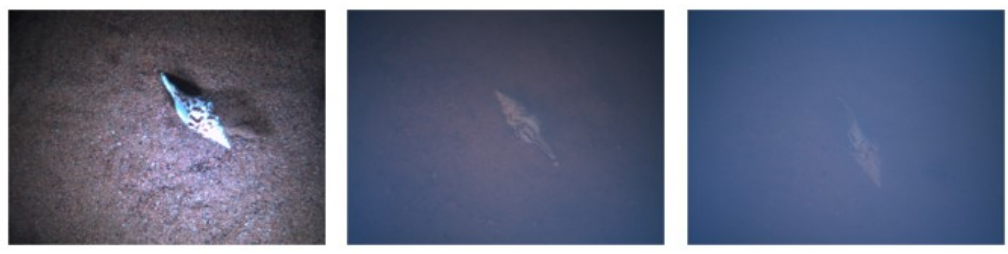

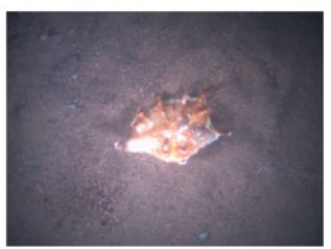

(a) Limpidity

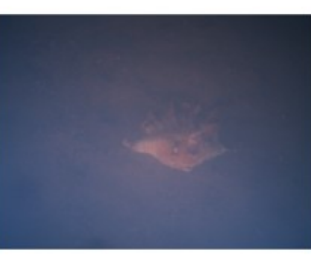

(b) Medium

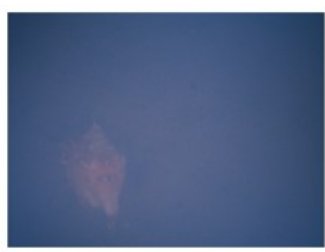

(c) High

Fig. 6. Underwater images captured with different degrees of water turbidity.

\subsection{Background Variations}

Although many saliency-detection algorithms have achieved great progress, their performance is still unsatisfactory when the images contain complex backgrounds, especially for those images captured from a complex and adverse underwater environment. Thus, background variations are essential for the database to assess and evaluate the performance of the underwater saliency-detection methods.

Six background variations, including coarse sand, medium sand, silver sand, and three degrees of variations in weed quantity (morsel, medium, and mickle), are considered in our underwater database. Fig. 7 shows some sample underwater images, under the six different backgrounds. 


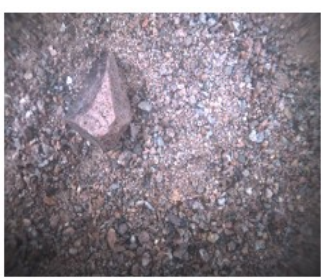

(a)

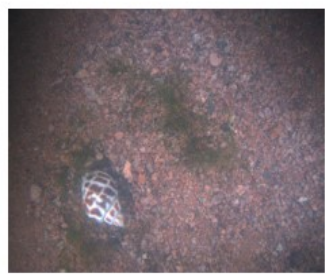

(d)

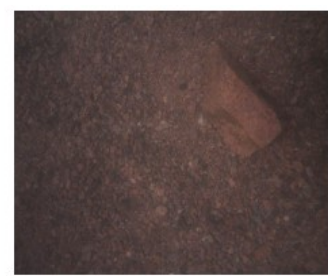

(b)

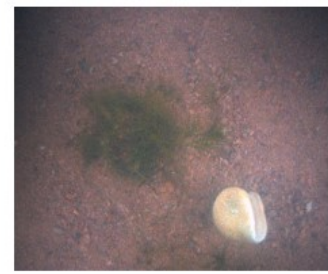

(e)

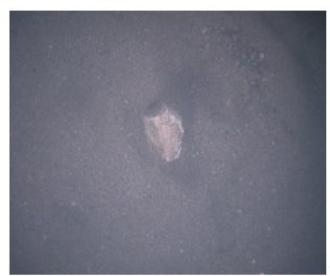

(c)

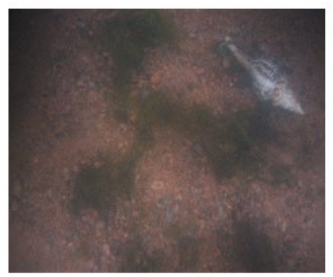

(f)

Fig. 7. Underwater objects at different background variations: (a) coarse sand, (b) medium sand, (c) silver sand, (d) a morsel of weed, (e) a medium amount of weed, and (f) a mickle of weed.

To reflect the real environments where an underwater object usually resides, we also take the floating debris into consideration in the construction of the underwater database. This will benefit the research on developing robust underwater saliency-detection algorithms for real applications under real underwater circumstances. Fig. 8 illustrates some examples of underwater salient objects, partly enveloped by some weed.
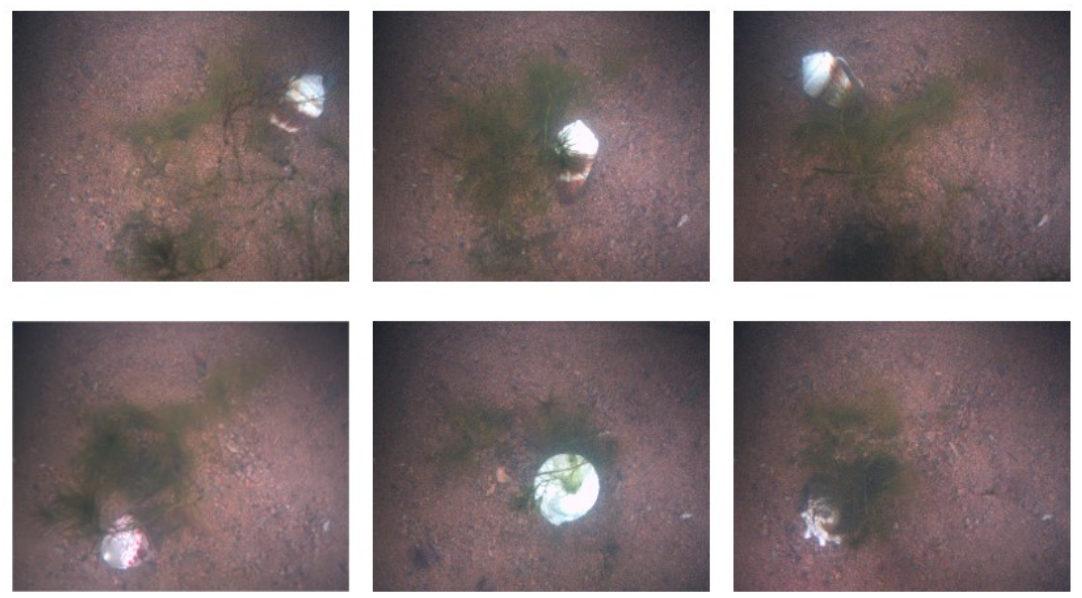

Fig. 8. Examples of underwater images, where the salient objects are partly enveloped by some weed.

\subsection{Multiple Salient Objects}


The number of salient objects in an image has an important influence on the saliency-detection results. In reality, multiple salient-object detection is a more challenging issue than the single salient-object detection. In order to evaluate the performance of an algorithm on multiple salient-object detection for underwater images, three different scenarios, namely a single salient object, two adjacent salient objects, and two detached salient objects, are included in the MUED. Fig. 9 shows some of the underwater images captured with a single salient object, two adjacent salient objects, and two detached salient objects.
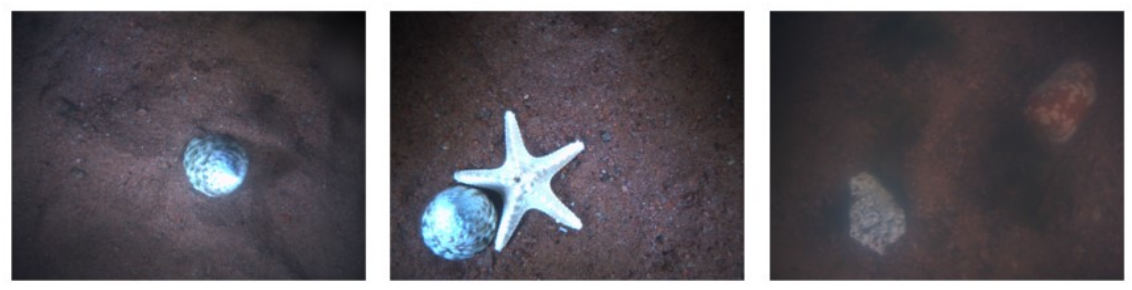

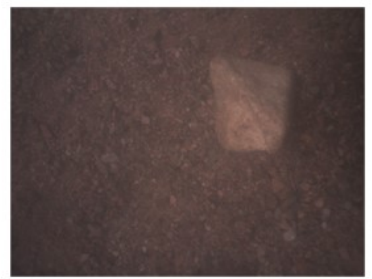

(a)

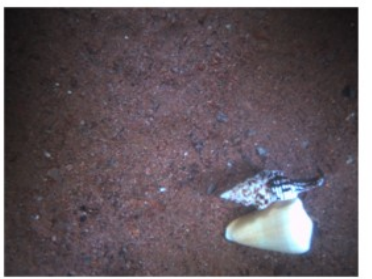

(b)

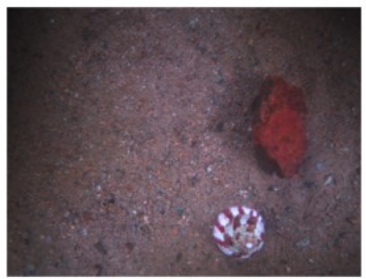

(c)

Fig. 9. Underwater images captured with (a) a single salient object, (b) two adjacent salient objects, and (c) two detached salient objects.

\subsection{Image Labeling}

The salient objects in the captured underwater images were manually labeled to form the ground-truth set. We have five participants for labeling the ground-truth set. They are postgraduate students with knowledge of object detection and saliency detection.

We cropped all the images to the size of $486 \times 648$ pixels (one quarter of the original size), retaining most of the important information in order to increase the speed of labeling. Each of the students was asked to draw a rectangle to specify the most salient object in each image, in the process of manually labeling the images. Each student spent about 30 seconds drawing a rectangle on an image, and entered the coordinates of the rectangle in a text file. In order to obtain accurate results, each person was asked to label one fifth of all the images independently, and check the labeling results done by the other four 
students. The whole labeling process took about two weeks for the five students. Fig. 10 shows some images with the labeled ground-truth information.
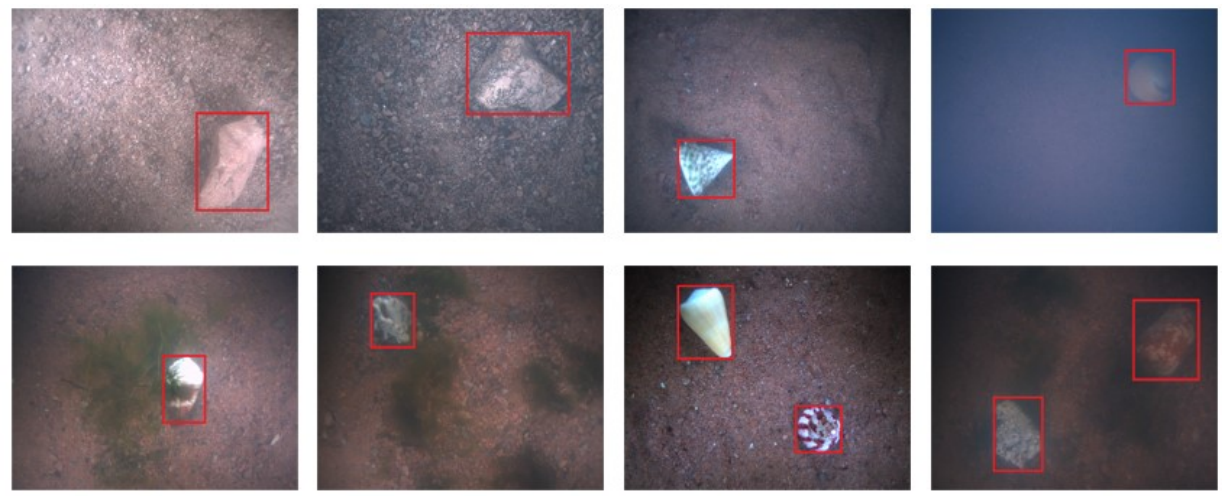

Fig. 10. Some example images with labeled ground-truth information, where each salient object is surrounded by a red rectangle.

\section{Publicly Released Marine Underwater Environment Database}

The MUED is now publicly accessible. It provides a useful resource for researchers who are working on underwater salient-object detection or saliency detection. This database provides researchers and the industry, working on underwater computer vision, with underwater images under different types of variations, especially pose, spatial location, illumination, turbidity of water, background, and multiple number of salient objects, in a uniform database. We will introduce this publicly released MUED in detail in this section.

\subsection{Contents of the Marine Underwater Environment Database}

The MUED contains 8600 underwater images of 430 distinct underwater objects. Each image has one or more salient objects, a relatively complex background and complicated variations in pose, location, illumination, and turbidity. Each object was captured in 20 images by considering four poses (described in Section 4.1) and five spatial locations (described in Section 4.2). To represent the environments that underwater images generally fall into, three degrees of water turbidity, four illumination variations, and seven background variations are also considered in the database. In addition, multiple salient objects and ground-truth information are included in this database for the research of more applicable and robust methods for both underwater salient-object detection and underwater computer vision. Thus, the released database has the advantages of containing a large quantity of underwater subjects, captured under different controlled variations. 


\subsection{Image Format}

The original 8600 underwater images are in RGB color format, with a resolution of $2592 \times 1944$ pixels each, captured by the camera MV-GE500C. The storage capacity required for the whole database is about 134.7 GB, which might cause a problem for researchers during processing. To alleviate this issue and retain most of the important information, we take the proportional scaling with a down-sample factor 1/4 into consideration. Thus, we scale down (reduce in resolution) all the images to the size of $648 \times 486$ pixels each, which leads the resolution to be one quarter of the original image.

\section{Evaluation on the Marine Underwater Environment Database}

The main purposes of evaluating baseline algorithms on the Marine Underwater Environment Database are as follows:

$\checkmark$ First, we want to assess the difficulty of saliency-detection algorithms on underwater images;

$\checkmark$ Second, we want to provide reference evaluation results for researchers working on underwater object detection and computer vision; and

$\checkmark$ Third, we want to identify the strengths and weaknesses of existing state-of-the-art saliencydetection algorithms for underwater images.

In the experiments, we compare nine different state-of-the-art methods, which were published in the past five years and achieved promising performance on normal images, including the GraphRegularization (GR) [34], Patch-Distinctness (PD) [35], Dense and Sparse Reconstruction (DSR) [36], Nonlinearly Covariance (NC) [37], Multi-Scale Superpixel (MSS) [38], Cellular Automata (CA) [39], Quaternionic Distance Based Weber Descriptor (QDWD) [18], Multiple Instance Learning (MIL) [40], and Pattern distinctness and Local contrast (PL) [41].

\subsection{Qualitative evaluation}

In order to qualitatively compare the nine state-of-the-art saliency-detection methods, Fig. 11 shows their saliency-detection results on the MUED. We can see that hardly any of these state-of-the-art saliency-detection algorithms can produce promising and satisfactory performance. The results, based on the PD, DSR and MSS methods, are lacking in some aspects, as some background regions are not suppressed completely and are falsely recognized as salient regions, which lead to the final saliency map containing much noise. These three methods mainly rely on low-level priors, which cannot achieve satisfactory performance when applied to underwater images with complicated illumination conditions, 
low contrast, turbidity of water, and lack of color information. Some methods, such as GR, NC and QDWD, produce inconspicuous boundaries, miss homogeneous regions of the underwater salient objects, and cannot even detect the salient objects completely, as illustrated in Fig. 3. The CA method, which detects salient objects based on cellular automata, is easily affected by illumination variations. This method erroneously detects the bright area and some backgrounds as salient regions. In addition, the turbidity of water has significant influence on the performance of the MIL method. This approach hardly distinguishes salient objects from the background when the underwater images are captured under the condition of severe turbidity of water. The PL method, which integrates directional stimuli, pattern distinctness, and local contrast to obtain the final saliency maps, can detect almost the entire set of salient objects, with obvious boundaries and less background pixels. Consequently, the PL method here produces slightly superior performance to other state-of-the-art saliency-detection algorithms. However, the background is not suppressed completely when the underwater images contains complicated lighting conditions, serious turbidity of water, and complex background. 

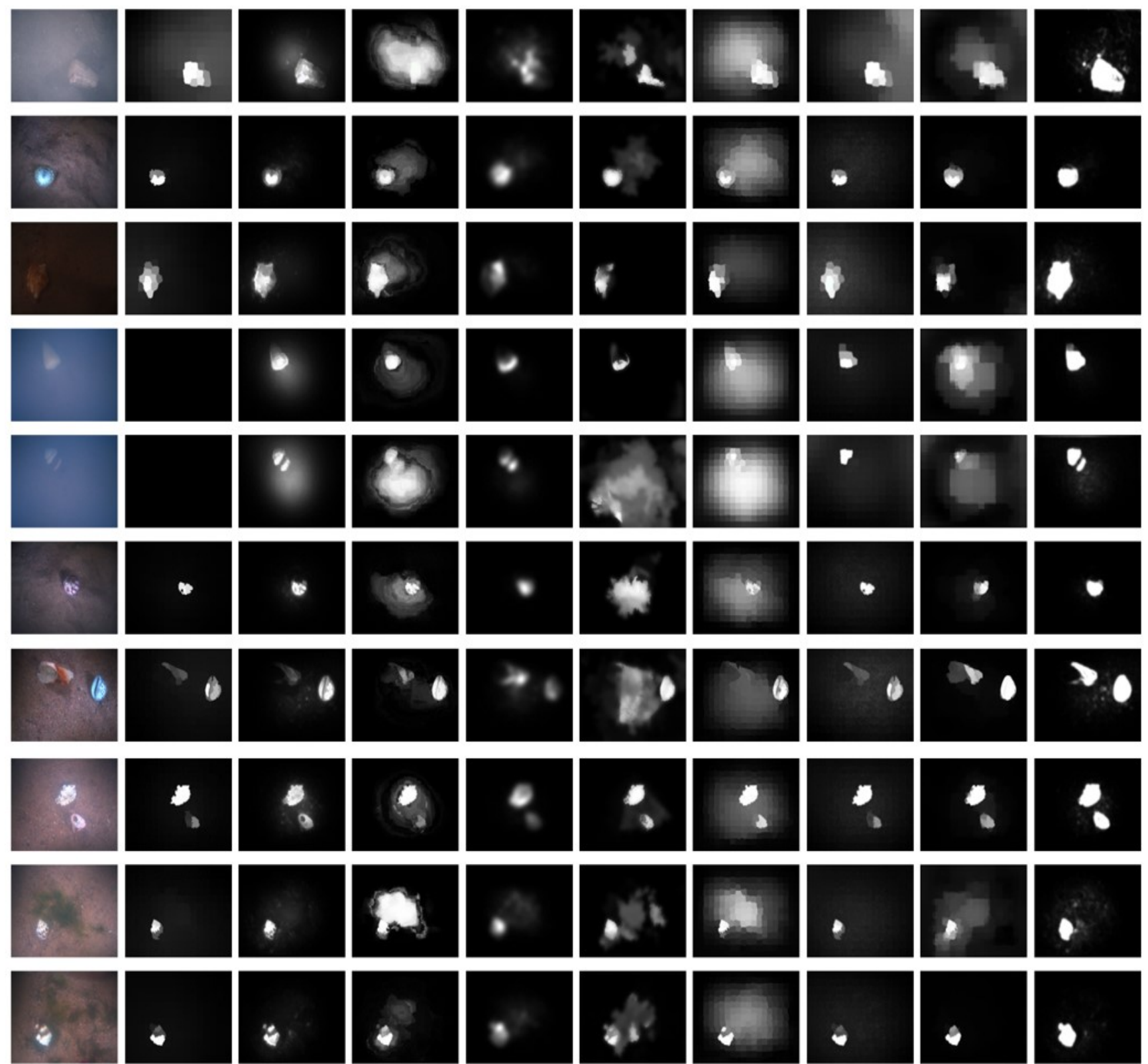

Input

GR

PD

DSR

NC

MSS

CA

QDWD

MIL

PL

Fig. 11. Some visual results based on the nine state-of-the-art saliency-detection algorithms.

\subsection{Quantitative evaluation}

To quantitatively assess the nine state-of-the-art saliency-detection methods, we now analyze the average Precision, Recall, F-measure and Receiver Operating Characteristics (ROC). The Precision measures the proportion of the detected salient-object rectangle which overlaps with the ground-truth rectangle, over the detected salient-object rectangle. The Recall is defined as the ratio of the detected 
salient-object rectangle overlap with the ground-truth rectangle, over the ground-truth rectangle. The $F$ measure is an overall evaluation, which provides a comprehensive measurement to weigh Precision more than Recall, and is defined as follows:

$$
F_{\beta}=\frac{(1+\beta) \times \text { Precision } \times \text { Recall }}{\beta \times \text { Precision }+ \text { Recall }},
$$

where $\beta$ is a real positive value, here set to 0.3 .

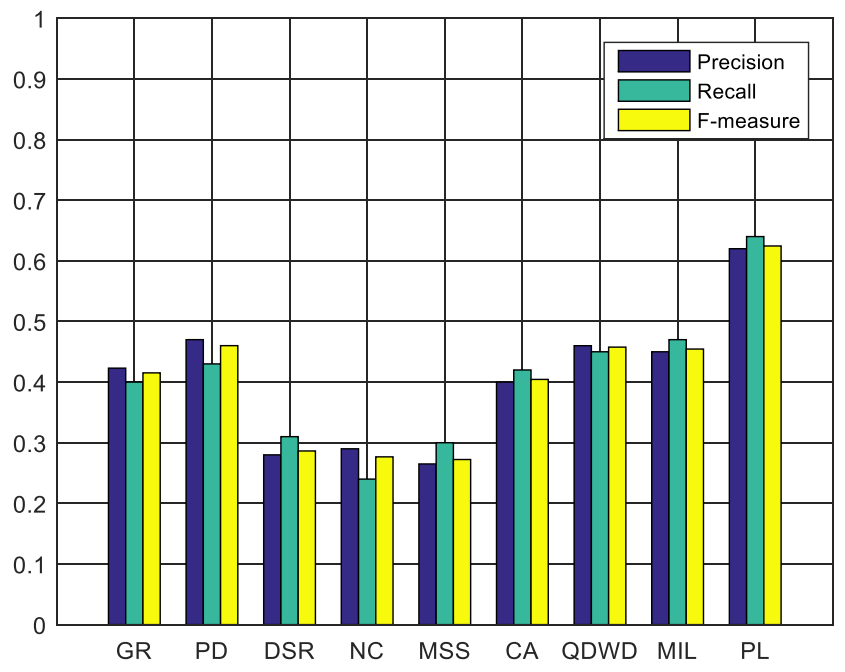

(a)

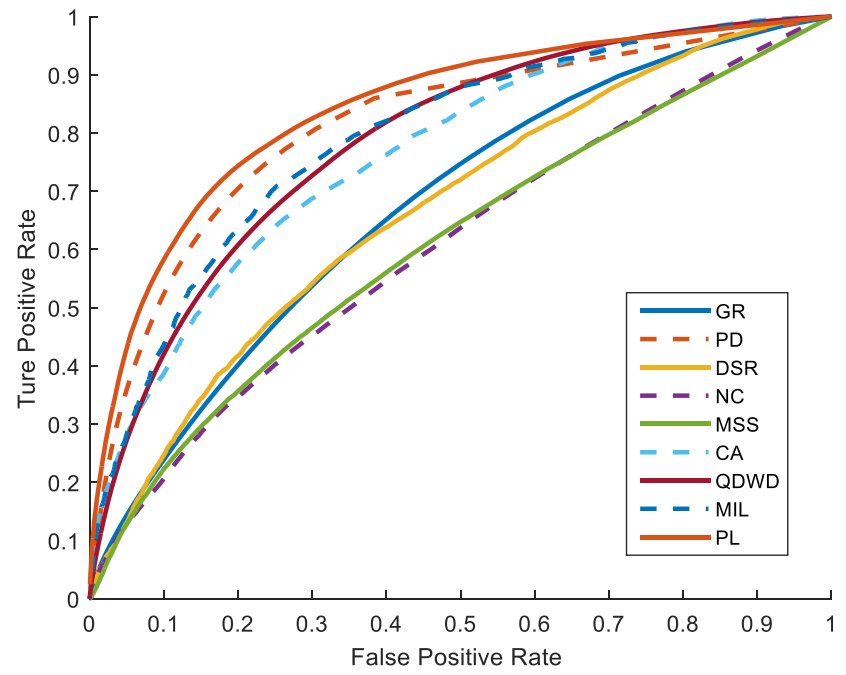

(b)

Fig. 12. Quantitative comparison of the nine saliency-detection methods on the MUED. (a) average Precision, Recall, and $F$-measure; (b) ROC curves.

Fig. 12 (a) shows the experimental results of the nine state-of-the-art methods, in terms of the average Precision, Recall and $F$-measure. For these three measures, we can see that all of the methods, except PL, have values lower than 50\%, which indicates that underwater image saliency detection is still a very challenging issue for existing methods. In underwater environments, the Recall rates of most methods are lower than 45\%, except for QDWD, MIL, and PL. For the Precision rate, only PD, QDWD and PL exceed 45\%. For the overall F- measure, only PD, QDWD, and PL achieve higher than 45\%, but lower than 50\%, except for PL, which scores over 60\%. The ROC curve is generated from the true-positive rate (TPR) and the false-positive rate (FPR) by altering the threshold, which is shown in Fig. 12 (b). According to the ROC curves, the PD and PL produce relatively superior performance to the other seven state-of-the-art algorithms. When comparing Fig. 12 to the results reported in literature obtained under much simpler conditions, we can observe that the performance of the methods in the complex 
underwater scenario is much worse. The experimental results also show the difficulty of saliency detection in the underwater vision domain.

Objects captured underwater are always blurred, like being surrounded by fog. Therefore, in this experiment, a defogging algorithm [53] is applied to preprocess the underwater images, and then the nine saliency-detection models are evaluated. We found that the resulting saliency maps are similar to those presented in Fig. 11, and the distinction between with or without preprocessing by the defogging algorithm is not obvious or conspicuous. Besides that, to compare the performance more precisely, we also compute the average Precision, Recall, $F$-measure and ROC curves of each saliency-detection method, with and without using defogging.

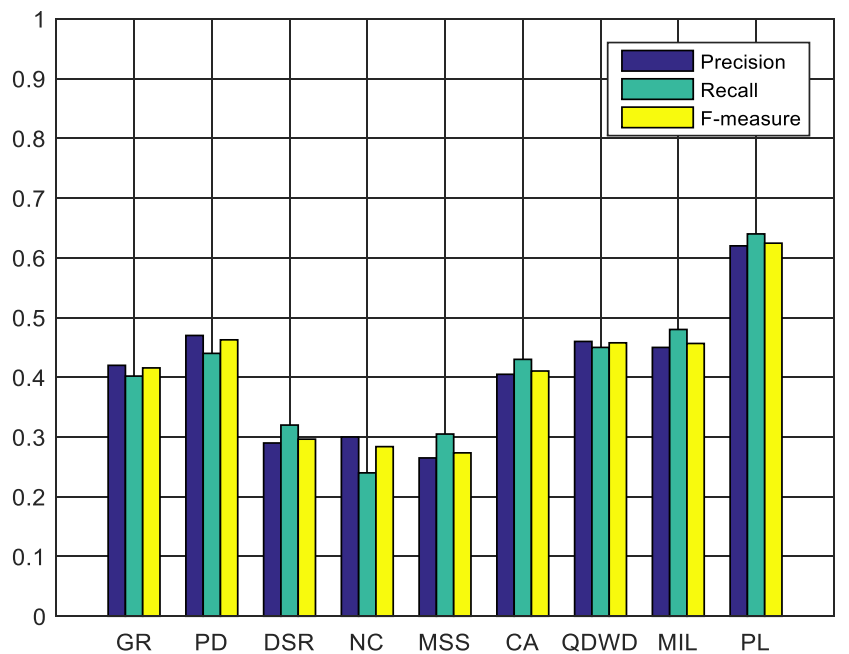

(a)

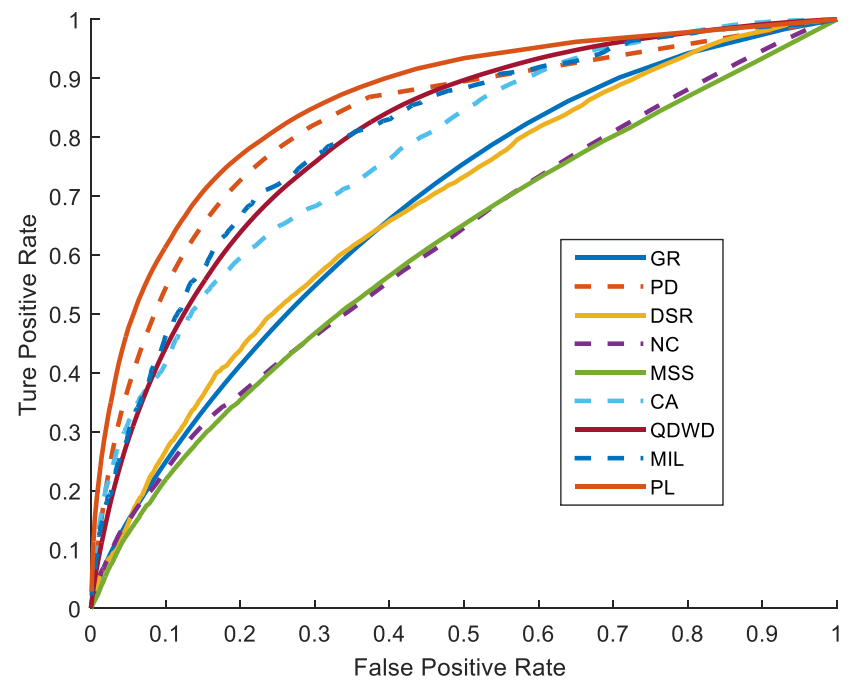

(b)

Fig. 13. Performance of nine state-of-the-art saliency-detection models based on the results of defogging preprocessing [53]. (a) average Precision, Recall, and F-measure; (b) ROC curves.

Fig. 13 (a) shows the experimental results of the nine state-of-the-art methods based on the results of defogging preprocessing [53], in terms of the average Precision, Recall, and $F$-measure. A comprehensive comparison, as shown in Figs. 12 (a) and 13 (a), indicates that the variations, as well as the effect of applying the defogging algorithm, are very small, ranging from -0.0024 to 0.0217 . Fig. 13 (b) shows the ROC curves of nine state-of-the-art methods based on the results of defogging preprocessing, which is very similar to Fig. 12 (b). All these experimental results show that applying a classic defogging algorithm cannot effectively improve saliency detection in underwater images. 
In addition, we also record the Mean Absolute Error (MAE) score, Overlapping Ratio (OR) score, and Area Under ROC Curve (AUC) score to further investigated the performance of the individual methods under different conditions. Since the challenge of the underwater saliency detection mainly comes from the lighting conditions, severe turbidity of water and complicated background, we separately discuss how each method behaves in different illumination, turbidity and background conditions for a comprehensive qualitative evaluation.

Tables 1-2 show the performance of each method in various illumination conditions. We find that each method performs better under high illumination than under low illumination. Meanwhile, Tables 3-4 tabulate the performance of individual method in various turbidity settings of the water, and demonstrate that the turbidity plays a key role on the accuracy of the underwater saliency detection. The reason is that the appearances of the underwater images are highly dependent on the turbidity of the water. The stronger the turbidity is, the more blurred and unclear the underwater images are, which pose a challenge for underwater saliency detection. Furthermore, Tables 5-6 show that the results of different saliency detection methods are better for a simple than for a complex background, which indicates that the complicated background of marine scenes is vital in underwater saliency detection. In brief, as tabulated in Tables 1-6, we can find that modeling underwater saliency-detection mechanism is still a tough and challenging task due to the variations in illumination, turbidity and complicated background conditions.

Table 1. Performance of different saliency-detection methods in high illumination.

\begin{tabular}{|c|c|c|c|c|c|c|c|c|c|}
\hline Methods & GR & PD & DSR & $\mathrm{NC}$ & MSS & CA & QDWD & MIL & PL \\
\hline MAE $\downarrow^{1}$ & 0.4971 & 0.3391 & 0.5867 & 0.6482 & 0.6433 & 0.4369 & $0.3390^{2}$ & 0.3407 & $0.2910^{2}$ \\
\hline $\mathrm{OR} \uparrow^{1}$ & 0.4819 & 0.5067 & 0.4603 & 0.4298 & 0.3952 & 0.3832 & 0.4944 & 0.5063 & 0.5264 \\
\hline $\mathbf{A U C} \uparrow$ & 0.5920 & 0.6212 & 0.5708 & 0.4218 & 0.4241 & 0.6072 & 0.6043 & 0.6419 & 0.6441 \\
\hline
\end{tabular}

${ }^{1}$ The $\uparrow$ indicates the larger value achieved, the better performance is, while the $\downarrow$ demonstrates the smaller, the better.

${ }^{2}$ The best top two results are highlighted with red and green fonts respectively.

Table 2. Performance of different saliency-detection methods in low illumination.

\begin{tabular}{c|c|c|c|c|c|c|c|c|c}
\hline \hline Methods & GR & PD & DSR & NC & MSS & CA & QDWD & MIL & PL \\
\hline MAE $\downarrow$ & 0.5228 & 0.4349 & 0.6058 & 0.6548 & 0.6743 & 0.4401 & 0.3492 & $\mathbf{0 . 3 4 8 1}$ & $\mathbf{0 . 3 1 9 2}$ \\
\hline OR $\uparrow$ & 0.4720 & 0.4829 & 0.4413 & 0.3981 & 0.3514 & 0.3433 & 0.4694 & $\mathbf{0 . 5 0 4 4}$ & $\mathbf{0 . 5 0 2 7}$ \\
\hline AUC $\uparrow$ & 0.5739 & 0.5413 & 0.5078 & 0.4025 & 0.3924 & 0.5722 & 0.5620 & $\mathbf{0 . 6 1 9 1}$ & $\mathbf{0 . 6 2 4 0}$ \\
\hline \hline
\end{tabular}


Table 3. Performance of different saliency-detection methods in limpid water.

\begin{tabular}{c|c|c|c|c|c|c|c|c|c}
\hline \hline Methods & GR & PD & DSR & NC & MSS & CA & QDWD & MIL & PL \\
\hline MAE $\downarrow$ & 0.5182 & 0.3734 & 0.5986 & 0.6489 & 0.6543 & 0.4399 & $\mathbf{0 . 3 2 9 9}$ & 0.3401 & $\mathbf{0 . 3 0 1 1}$ \\
\hline OR $\uparrow$ & 0.4804 & $\mathbf{0 . 5 0 6 6}$ & 0.4601 & 0.4184 & 0.3795 & 0.3683 & 0.4912 & 0.4384 & $\mathbf{0 . 5 0 6 2}$ \\
\hline AUC $\uparrow$ & 0.5890 & 0.6141 & 0.5690 & 0.4205 & 0.4122 & 0.5937 & 0.6022 & $\mathbf{0 . 6 2 4 1}$ & $\mathbf{0 . 6 3 3 4}$ \\
\hline \hline
\end{tabular}

Table 4. Performance of different saliency-detection methods in turbid water.

\begin{tabular}{c|c|c|c|c|c|c|c|c|c}
\hline \hline Methods & GR & PD & DSR & NC & MSS & CA & QDWD & MIL & PL \\
\hline MAE $\downarrow$ & 0.5281 & 0.4340 & 0.6068 & 0.6844 & 0.6843 & 0.5369 & 0.4491 & $\mathbf{0 . 3 7 6 2}$ & $\mathbf{0 . 3 8 9 9}$ \\
\hline OR $\uparrow$ & 0.4214 & $\mathbf{0 . 4 9 3 6}$ & 0.4103 & 0.3982 & 0.3359 & 0.3383 & 0.4394 & 0.4311 & $\mathbf{0 . 4 9 8 9}$ \\
\hline AUC $\uparrow$ & 0.5697 & 0.5834 & 0.5271 & 0.3985 & 0.3911 & 0.5721 & $\mathbf{0 . 5 9 4 2}$ & 0.5919 & $\mathbf{0 . 5 9 8 3}$ \\
\hline \hline
\end{tabular}

Table 5. Performance of different saliency-detection methods with simple background.

\begin{tabular}{c|c|c|c|c|c|c|c|c|c}
\hline \hline Methods & GR & PD & DSR & NC & MSS & CA & QDWD & MIL & PL \\
\hline MAE $\downarrow$ & 0.5028 & 0.4303 & 0.5886 & 0.6486 & 0.6483 & 0.4396 & $\mathbf{0 . 3 4 0 1}$ & 0.3422 & $\mathbf{0 . 3 0 2 0}$ \\
\hline OR $\uparrow$ & 0.4920 & $\mathbf{0 . 4 9 3 7}$ & 0.4603 & 0.4298 & 0.3915 & 0.3812 & 0.4901 & 0.4309 & $\mathbf{0 . 5 0 0 2}$ \\
\hline AUC $\uparrow$ & 0.5897 & 0.6014 & 0.5499 & 0.4200 & 0.4004 & 0.5972 & 0.6042 & $\mathbf{0 . 6 1 9 4}$ & $\mathbf{0 . 6 2 0 4}$ \\
\hline \hline
\end{tabular}

Table 6. Performance of different saliency-detection methods with complex background.

\begin{tabular}{c|c|c|c|c|c|c|c|c|c}
\hline \hline Methods & GR & PD & DSR & NC & MSS & CA & QDWD & MIL & PL \\
\hline MAE $\downarrow$ & 0.5128 & 0.4324 & 0.6056 & 0.6880 & 0.6833 & 0.5436 & 0.4429 & $\mathbf{0 . 3 9 2 7}$ & $\mathbf{0 . 3 7 2 0}$ \\
\hline OR $\uparrow$ & 0.4782 & $\mathbf{0 . 4 7 9 8}$ & 0.4432 & 0.4189 & 0.3556 & 0.3830 & 0.4394 & 0.4218 & $\mathbf{0 . 4 8 5 2}$ \\
\hline AUC $\uparrow$ & 0.5541 & 0.5984 & 0.5044 & 0.4022 & 0.3924 & 0.5729 & 0.5420 & $\mathbf{0 . 6 0 9 8}$ & $\mathbf{0 . 6 0 9 0}$ \\
\hline \hline
\end{tabular}

\subsection{Generalization analysis on the Marine Underwater Environment Database}

In recent years, some state-of-the-art methods have been proposed to improve the performance for saliency detection and excellent progress has been made when dealing with simple images. However, applying the saliency-detection mechanism to underwater scenes is a new research direction and still a challenging task. Most of the state-of-the-art saliency-detection models scarcely adapts to the complex underwater environments. The failure of existing saliency-detection algorithms is mainly caused by the complex underwater environment in terms of the following aspects:

variations of complicated lighting conditions,

low-contrast objects with the background, 
light absorption and scattering in water,

complex seabed environments with influence of water plants,

severe turbidity of the water, and

lack of color information of underwater objects.

All of these effects often occur in combination and can interact mutually, which forms an open and challenging problem to be solved by underwater saliency-detection algorithms. In other words, further efforts for underwater saliency detection must be made for the future development of underwater computer vision.

\section{Obtain the Marine Underwater Environment Database}

We have made the data available in a public repository without any password protection, the information on how to freely obtain a copy of the MUED can be found on the following website, Database Part A: http://doi.org/10.5281/zenodo.2542305; Database Part B: http://doi.org/10.5281/zenodo.2542307.

\section{Conclusion and Discussion}

In this paper, we have introduced the Marine Underwater Environment Database (MUED) and the detail of the experiment set-up. The released MUED contains 8600 underwater images of 430 underwater objects, with different types of variations, especially pose, spatial location, illumination, turbidity of water, multiple salient objects, and diverse backgrounds. Manually labeled ground-truth information is also included in the underwater database, which makes it very useful for the research of more applicable and robust methods in both underwater salient-object detection and underwater computer vision. The diverse underwater image database, which contains a large quantity of distinct objects captured under different underwater environments, offers unparalleled opportunities to researchers working on underwater computer vision. A number of the state-of-the-art saliency-detection algorithms have also been evaluated on the database. Experimental results show that existing algorithms face a great challenge when applying to underwater images.

\section{Acknowledgments}

This work was supported by National Natural Science Foundation of China (NSFC) (61601427, 61602229, 61771230); Royal Society - K. C. Wong International Fellow; Natural Science Foundation of Shandong Province (ZR2016FM40); Shandong Provincial Key Research and Development Program of 
China (NO. 2017CXGC0701); Fostering Project of Dominant Discipline and Talent Team of Shandong Province Higher Education Institutions.

\section{REFERENCES}

[1] Lu, W., Hou, J., Yan, Y. et al., "MSQL: efficient similarity search in metric spaces using SQL", The VLDB Journal (2017) 26: 829. https://doi.org/10.1007/s00778-017-0481-6.

[2] Belesiotis, A., Skoutas, D., Efstathiades, C. et al., "Spatio-textual user matching and clustering based on set similarity joins", The VLDB Journal (2018) 27: 297. https://doi.org/10.1007/s00778-018-0498-5.

[3] Y. Ding, J. Xiao, and J. Yu, "Importance filtering for image retargeting," In: Computer Vision and Pattern Recognition, pp. 89-96, 2011.

[4] Li, Y., U, L.H., Yiu, M.L. et al., "Efficient discovery of longest-lasting correlation in sequence databases", The VLDB Journal (2016) 25: 767. https://doi.org/10.1007/s00778-016-0432-7.

[5] A. Santella, M. Agrawala, D. Decarlo, et al, "Gaze-based interaction for semi-automatic photo cropping," SIGCHI, pp. 771-780, 2006.

[6] C. Siagian, L. Itti, "Rapid biologically-inspired scene classification using features shared with visual attention," IEEE transactions on pattern analysis and machine intelligence, vol. 29, no. 2, pp. 300-312, 2007.

[7] L. Itti, C. Koch, E. Niebur, "A model of saliency based visual attention for rapid scene analysis," IEEE Transactions on pattern analysis and machine intelligence, vol. 20, no. 11, pp. 1254-1259, 1998.

[8] F Perazzi, P Krähenbühl, Y Pritch, et al, "Saliency filters: Contrast based filtering for salient region detection”. In Computer Vision and Pattern Recognition, pp. 733-740, 2012.

[9] A. Borji, "What is a salient object? a dataset and a baseline model for salient object detection," IEEE Transactions on Image Processing, vol. 24, no. 2, pp. 742-756, 2015.

[10] C. Yang, L. Zhang, H. Lu, X. Ruan, et al, "Saliency detection via graph-based manifold ranking," IEEE conference on computer vision and pattern recognition, pp. 3166-3173, 2013.

[11] J. Harel, C. Koch, P. Perona, "Graph-based visual saliency," Advances in neural information processing systems, pp. 545-552, 2006.

[12] X. Hou, L. Zhang, "Saliency detection: a spectral residual approach," IEEE conference on computer vision and pattern recognition, pp. 1-8, 2007.

[13] Y. F. Ma, H. J. Zhang, "Contrast-based image attention analysis by using fuzzy growing," ACM conference on Multimedia, pp. 374-381, 2003. 
[14] N. Murray, M. Vanrell, X. Otazu, et al, "Saliency estimation using a non-parametric low-level vision model," IEEE conference on computer vision and pattern recognition, pp. 433-440, 2011.

[15] M. Jian, K. M. Lam, J. Dong, et al, "Visual-patch-attention-aware Saliency Detection," IEEE transactions on cybernetics, vol. 45, no. 8, pp. 1575-1586, 2015.

[16] H. Song, Z. Liu, Y. Xie, et al, "RGBD Co-saliency Detection via Bagging-Based Clustering," IEEE Signal Processing Letters, vol. 23, no. 12, pp. 1707-1711, 2016.

[17] M. Jian, Q. Qi, Y. Sun, K-M Lam, "Saliency Detection Using Quaternionic Distance Based Weber Descriptor and Object Cues," Signal and Information Processing Association Annual Summit and Conference, pp. 1-4, 2016.

[18] M. Jian, Q. Qi, J. Dong, K-M Lam, "Saliency detection using quaternionic distance based weber local descriptor and level priors," Multimedia Tools and Applications, pp. 1-18, 2017.

[19]H. Lei, H. Xie, W. Zou, et al, "Hierarchical Saliency Detection via Probabilistic Object Boundaries," International Journal of Pattern Recognition and Artificial Intelligence, vol. 31, no. 06, 2017.

[20] C. Kanan, M. H. Tong, L. Zhang, et al, "SUN: Top-down saliency using natural statistics," Visual Cognition, vol. 17, no.6-7, pp. 979-1003, 2009.

[21] J. Yang, M. H. Yang, “Top-down visual saliency via joint CRF and dictionary learning," IEEE conference on computer vision and pattern recognition, pp. 2293-2303, 2012.

[22] F. Murabito, C. Spampinato, S. Palazzo, et al, “Top-Down Saliency Detection Driven by Visual Classification" arXiv preprint arXiv:1709.05307, 2017.

[23] S. He, R. W. Lau, Q. Yang, "Exemplar-driven top-down saliency detection via deep association," Proceedings of the IEEE Conference on Computer Vision and Pattern Recognition, pp. 5723-5732, 2016.

[24] L. Wang, H. Lu, X. Ruan, M.-H. Yang, “Deep networks for saliency detection via local estimation and global search," IEEE conference on computer vision and pattern recognition, pp. 3183-3192, 2015.

[25] G. Li, Y. Yu, “Deep contrast learning for salient object detection,” IEEE conference on computer vision and pattern recognition, pp. 478-487, 2016.

[26] X. Li, L. Zhao, L. Wei, et al, “Deep saliency: Multi-task deep neural network model for salient object detection,” IEEE Transactions on Image Processing, vol. 25, no. 8, pp. 3919-3930, 2016.

[27] Q. Hou, M. M. Cheng, X. W. Hu, et al, "Deeply supervised salient object detection with short connections," arXiv preprint arXiv:1611.04849, 2016. 
[28] P. Zhang, D. Wang, H. Lu, et al, "Learning Uncertain Convolutional Features for Accurate Saliency Detection,” arXiv preprint arXiv:1708.02031, 2017.

[29] G. L. Foresti, S. Gentili, "A vision based system for object detection in underwater images," International Journal of Pattern Recognition and Artificial Intelligence, vol. 14, no. 02, pp. 167-188, 2000.

[30] T. D. Zhang, L. Wan, et al, "Underwater image detection based on the discrete fractional Brownian random field," Opto-Electronic Engineering, vol. 35, no. 8, pp. 41-46, 2008.

[31] M. Dubreuil, P. Delrot, et al, "Exploring underwater target detection by imaging polarimetry and correlation techniques," Applied optics, vol. 52, no. 5, pp. 997-1005, 2013.

[32] H. B. Wang, X. Dong, J. Shen, et al, "Salieney based adaptive object extraction for color underwater images," Applied Mechanics and Materials, pp. 2651-2655, 2013.

[33] M. Jian, Q. Qi, J. Dong, et al, "The OUC-vision large-scale underwater image database," 2017 IEEE International Conference on Multimedia and Expo (ICME), pp. 1297-1302, 2017.

[34] C. Yang, L. Zhang, H. Lu, "Graph-regularized saliency detection with convex-hull-based center prior,” IEEE Signal Processing Letters, vol. 20, no. 7, pp. 637-640, 2013.

[35] R. Margolin, A. Tal, and L. Zelnik-Manor, "What makes a patch distinct?" IEEE conference on computer vision and pattern recognition, pp. 1139-1146, 2013.

[36] X. Li, H. Lu, L. Zhang, X. Ruan, et al, "Saliency detection via dense and sparse reconstruction," Proceedings of the IEEE International Conference on Computer Vision, pp. 2976-2983, 2013.

[37] E. Erdem and A. Erdem, "Visual saliency estimation by nonlinearly integrating features using region covariances," Journal of vision, vol. 13, no. 4, pp. 11, 2013.

[38] N. Tong, H. Lu, L. Zhang, et al, "Saliency detection with multi-scale superpixels," IEEE Signal Processing Letters, vol. 21, no. 9, pp. 1035-1039, 2014.

[39] Y. Qin, H. Lu, Y. Xu, et al, "Saliency detection via cellular automata," Proceedings of the IEEE Conference on Computer Vision and Pattern Recognition, pp. 110-119, 2015.

[40] F. Huang, J. Qi, H. Lu, et al, "Salient Object Detection via Multiple Instance Learning," IEEE Transactions on Image Processing, vol. 26, no. 4, pp. 1911-1922, 2017.

[41] M. Jian, Q. Qi, J. Dong, et al, "Integrating QDWD with pattern distinctness and local contrast for underwater saliency detection," Journal of Visual Communication and Image Representation, vol. 53, pp. 31-41, 2018. 
[42] Amanda Duarte, Felipe Codevilla, et. Al. A dataset to evaluate underwater image restoration methods, Conference: OCEANS 2016-Shanghai, DOI: 10.1109/OCEANSAP.2016.7485524.

[43] Y. Pang, L. Ye, X. Li, et al, "Incremental learning with saliency map for moving object detection," IEEE Transactions on Circuits and Systems for Video Technology, vol. 28, no. 3, pp. 640-651, 2018. [44] Y. Pang, H. Zhu, X. Li, et al, "Motion blur detection with an indicator function for surveillance machines," IEEE Transactions on Industrial Electronics, vol. 63, no. 9, pp. 5592-5601, 2016.

[45] Y. Pang, M. Sun, X. Jiang, et al, "Convolution in convolution for network in network," IEEE Transactions on Neural Networks and Learning Systems, vol. 29, no. 5, pp. 1587-1597, 2018.

[46] J. Jaffe, K. Moore, J. McLean, et al, "Underwater optical imaging: status and prospects," Oceanography, vol. 14, no. 3, pp. 66-76, 2001.

[47] Y. Schechner, N. Karpel, "Clear underwater vision," Computer Vision and Pattern Recognition, 2004. CVPR 2004. Proceedings of the 2004 IEEE Computer Society Conference on. IEEE, vol. 1, pp. II, 2003.

[48] R. Schettini, S. Corchs, "Underwater image processing: state of the art of restoration and image enhancement methods," EURASIP Journal on Advances in Signal Processing, 2010(1): 746052.

[49] J. Chiang, Y. Chen, "Underwater image enhancement by wavelength compensation and dehazing," IEEE Transactions on Image Processing, vol. 21, no. 4, pp. 1756-1769, 2012.

[50] C. Li, J. Guo, R. Cong, et al, "Underwater image enhancement by dehazing with minimum information loss and histogram distribution prior," IEEE Transactions on Image Processing, vol. 25, no. 12, pp. 5664-5677, 2016.

[51] Y. Peng, P. Cosman, "Underwater image restoration based on image blurriness and light absorption," IEEE Transactions on Image Processing, vol. 26, no, 4, pp. 1579-1594, 2017.

[52] M. Mangeruga, M. Cozza, F. Bruno, "Evaluation of Underwater Image Enhancement Algorithms under Different Environmental Conditions," Journal of Marine Science and Engineering, vol. 6, no. 1, pp. 10, 2018.

[53] K. He, J. Sun, X. Tang, "Single image haze removal using dark channel prior," IEEE transactions on pattern analysis and machine intelligence, vol. 33, no. 12, pp. 2341-2353, 2011.

[54] T. Weise, X. Wang, Q. Qi, B. Li, K. Tang. Automatically discovering clusters of algorithm and problem instance behaviors as well as their causes from experimental data, algorithm setups, and instance features. Applied Soft Computing, 73:366-382, 2018. 


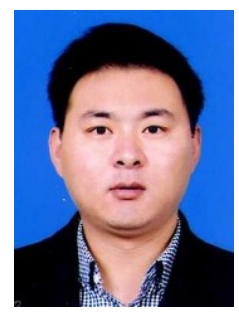

Muwei Jian received the $\mathrm{PhD}$ degree from the Department of Electronic and Information Engineering, The Hong Kong Polytechnic University, in October 2014. He was a Lecturer with the Department of Computer Science and Technology, Ocean University of China, from 2015 to 2017. Currently, Dr. Jian is a Professor and Ph.D Supervisor at the School of Computer Science and Technology, Shandong University of Finance and Economics.

His current research interests include human face recognition, image and video processing, machine learning and computer vision. Prof. Jian was actively involved in professional activities. He has been a member of the Program Committee and Special Session Chair of several international conferences, such as SNPD 2007, ICIS 2008, APSIPA 2015, EEECS 2016, ICTAI2016, ICGIP 2016 and ICTAI 2017. Dr. Jian has also served as a reviewer for several international SCI-indexed journals, including IEEE Trans., Pattern Recognition, Information Sciences, Computers in Industry, Machine Vision and Applications, Machine Learning and Cybernetics, The Imaging Science Journal, and Multimedia Tools and Applications.

Prof. Jian holds 3 granted national patents and has published over 40 papers in refereed international leading journals/conferences such as IEEE Trans. on Cybernetics, IEEE Trans. on Circuits and Systems for Video Technology, Pattern Recognition, Information Sciences, Signal Processing, ISCAS, ICME and ICIP.

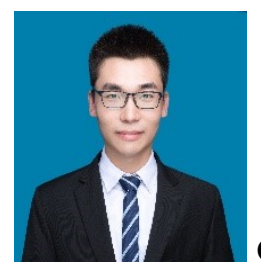

Qiang Qi is pursuing his Master's degree at the Department of Computer Science and Technology, Ocean University of China. His research interests include image processing, pattern recognition, and underwater vision.

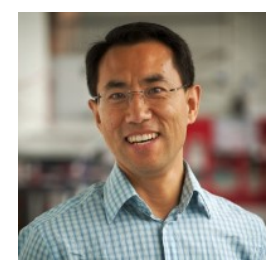

Hui Yu is currently a professor in the School of Creative Technologies. Dr. Yu is the leader of Visual Computing Group at the University of Portsmouth. He previously held an appointment with the University of Glasgow. He has won prizes for his study and research including Excellent Undergraduate Prize (provincial level), the Best PhD Thesis Prize, EPSRC DHPA Awards (PhD) and Vice Chancellor Travel Prize. Dr. Yu is an Associate Editor of IEEE Transactions on Human-Machine Systems. He is a member of the Peer Review College of the Engineering and Physical Sciences Research Council (EPSRC), UK. 
Junyu Dong received his B.Sc. and M.Sc. in Applied Mathematics from the Ocean University of China (formerly called Ocean University of Qingdao) in 1993 and 1999, respectively. He won the Overseas Research Scholarship and James Watt Scholarship for his PhD study in 2000 and was awarded a Ph.D. degree in Image Processing in 2003 from the School of Mathematical and Computer Sciences, Heriot-Watt University, UK.

Dr. Junyu Dong joined Ocean University of China in 2004. From 2004 to 2010, Dr. Junyu Dong was an associate professor at the Department of Computer Science and Technology. He became a Professor in 2010 and is currently the Head of the Department of Computer Science and Technology. Prof. Dong was actively involved in professional activities. He has been a member of the program committee of several international conferences, including the 4th International Workshop on Texture Analysis and Synthesis (associated with ICCV2005), the 2006 British Machine Vision Conference (BMVC 2006) and the 3rd International Conference on Appearance (Predicting Perceptions 2012). Currently, Prof. Dong is the Chairman of Qingdao Young Computer Science and Engineering Forum (YOCSEF Qingdao). He is a member of ACM and IEEE. Prof. Dong's research interest includes texture perception and analysis, 3D reconstruction, video analysis and underwater image processing.

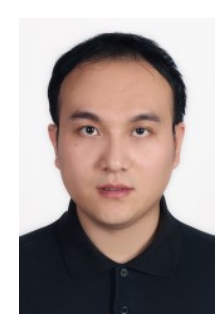

Chaoran Cui received the B.S. degree in Software Engineering in 2010 and the Ph.D. degree in Computer Science and Technology in 2015, both from Shandong University, Jinan, China. During 2015-2016, he was a research fellow at Singapore Management University. He is now a Professor in School of Computer Science and Technology at Shandong University of Finance and Economics. His research interests include information retrieval, analysis and understanding on multimedia information, and computer vision.

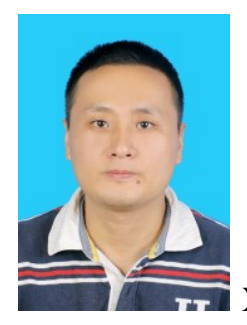

Xiushan Nie received the Ph.D. degree from Shandong University, Shandong, China, in 2011. He is currently a Professor with Shandong University of Finance and Economics, Shandong, China. From 2013 to 2014, he was a Visiting Scholar with the University of Missouri-Columbia, Columbia, MO,USA. His research interests include multimedia retrieval and indexing, multimedia security, and computer vision. 
Huaxiang Zhang is currently a professor with the School of Information Science and Engineering \& the Institute of Data Science and Technology, Shandong Normal University, China. He received his Ph.D. from Shanghai Jiaotong University in 2004, and worked as an associated professor with the Department of Computer Science, Shandong Normal University from 2004 to 2005 . He has authored over 180 journal and conference papers and holds 13 granted national patents. His current research interests include machine learning, pattern recognition, evolutionary computation, cross-media retrieval, web information processing, big data analysis, etc.

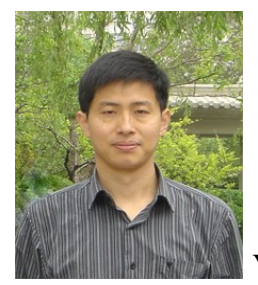

Yilong Yin received the Ph.D. degree from Jilin University, Changchun, China, in 2000. From 2000 to 2002, he was a Post-Doctoral Fellow with the Department of Electronics Science and Engineering, Nanjing University, Nanjing, China.

$\mathrm{He}$ is currently the Director of the data Mining, Machine Learning, and their Applications Group and a Professor of the School of Computer Science and Technology, Shandong University, Jinan, China. His research interests include machine learning, data mining, and computational medicine.

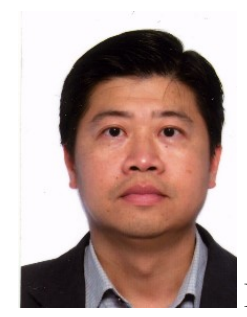

Kin-Man Lam received the Associateship in Electronic Engineering with distinction from The Hong Kong Polytechnic University (formerly called Hong Kong Polytechnic) in 1986, the M.Sc. degree in communication engineering from the Department of Electrical Engineering, Imperial College of Science, Technology and Medicine, London, U.K., in 1987, and the Ph.D. degree from the Department of Electrical Engineering, University of Sydney, Sydney, Australia, in August 1996.

From 1990 to 1993, Prof. Lam was a lecturer at the Department of Electronic Engineering of The Hong Kong Polytechnic University. He joined the Department of Electronic and Information Engineering, The Hong Kong Polytechnic University again as an Assistant Professor in October 1996. He became an Associate Professor in 1999, and is now a 
Professor. Prof. Lam was actively involved in professional activities. He has been a member of the organizing committee or program committee of many international conferences. In particular, he was the Secretary of the 2003 IEEE International Conference on Acoustics, Speech, and Signal Processing (ICASSP'03), the Technical Chair of the 2004 International Symposium on Intelligent Multimedia, Video and Speech Processing (ISIMP 2004), a Technical Co-Chair of the 2005 International Symposium on Intelligent Signal Processing and Communication Systems (ISPACS 2005), a secretary of the 2010 International Conference on Image Processing (ICIP 2010), a Technical Co-Chair of 2010 Pacific-Rim Conference on Multimedia (PCM 2010), and a General Co-Chair of the 2012 IEEE International Conference on Signal Processing, Communications, \& Computing (ICSPCC 2012), which was held in Hong Kong in August 2012. He was a General Co-Chair of the 2017 IEEE International Conference on Multimedia and Expo (ICME 2017). Prof. Lam was the Chairman of the IEEE Hong Kong Chapter of Signal Processing between 2006 and 2008.

Currently, he is the VP-Member Relations and Development of the Asia-Pacific Signal and Information Processing Association (APSIPA) and the Director-Student Services of the IEEE Signal Processing Society. Prof. Lam serves as an Associate Editor of IEEE Trans. on Image Processing, Digital Signal Processing, APSIPA Trans. on Signal and Information Processing, and EURASIP International Journal on Image and Video Processing. He is also an Editor of HKIE Transactions and an Area Editor of the IEEE Signal Processing Magazine. His current research interests include human face recognition, image and video processing, and computer vision. 